Pacific Journal of Mathematics

THE SUM OF THE DISTANCES TO CERTAIN POINTSETS ON 


\title{
THE SUM OF THE DISTANCES TO CERTAIN POINTSETS ON THE UNIT CIRCLE
}

\author{
KenNeth B. Stolarsky
}

Let $p_{1}, \cdots, p_{n}$ be points on the unit circle $U$. For $p \in U$ define $T_{\lambda}(p)=\Sigma\left|p-p_{i}\right|^{\lambda}$. The extremal behavior of $T_{\lambda}(p)$ is completely described in two cases: (i) $0 \leqq \lambda \leqq 2$ and $n=3$ and (ii) $0 \leqq \lambda<2 n$ and $p_{1}, \cdots, p_{n}$ the vertices of a regular n-gon.

Introduction. Let $|p-q|$ denote the Euclidean distance between $p$ and $q$. The first main result of this paper (proved in $\S 5$ ) is

THEOREM 1.1. If $p_{1}, p_{2}$, and $p_{3}$ are points on the unit circle $U$, and $0 \leqq \lambda \leqq 2$, then there is $a p \in U$ such that

$$
\sum_{i=1}^{3}\left|p-p_{i}\right|^{2} \geqq 2+2^{2},
$$

and this is best possible.

The second main result (proved in $\S 2$ ) is

THEOREM 1.2. Let $e_{1}, \cdots, e_{n}$ be the vertices of a regular n-gon. Let $p \in U$, and for $0 \leqq \lambda<2 n$ let

$$
T_{\lambda}(p)=\sum_{i=1}^{n}\left|p-e_{i}\right|^{\lambda} .
$$

If $\lambda$ is an even integer, then $T_{\lambda}(p)$ is constant. Otherwise, let $m$ be the integer such that

$$
2 m<\lambda<2(m+1)
$$

If $m$ is even (odd), then $T_{\lambda}(p)$ is maximal (minimal) if and only if $p$ bisects the arc between consecutive $e_{i}$. Moreover, $T_{\lambda}(p)$ is minimal (maximal) if and only if $p=e_{i}$ for some $i$.

It follows from the case $n=3$ of Theorem 1.2 that Theorem 1.1 is best possible. Theorem 1.2 is proved by means of a differential inequality related to Sturm-Liouville problems [3, pp. 140-142]. A more restricted version of this theorem is proved in [11] by means of an integral transform.

We remark that in many extremal problems of this type the cases $0 \leqq \lambda \leqq 1$ and $1<\lambda \leqq 2$ tend to be different, or at least require 
different methods. For example, the problem of placing points $p_{1}$, $\cdots, p_{n}$ on the unit circle so that

$$
S(n)=\sum_{i<j}\left|p_{i}-p_{j}\right|^{\lambda}
$$

is maximal has been solved for $0 \leqq \lambda \leqq 1$; one simply places them at the vertices of a regular $n$-gon $[2,6]$. However, for $1<\lambda<2$ this is still an open question. We comment that there is a growing literature on the sum (1.4) and its analogues $S(n, m)$ in $m$-dimensional Euclidean space; see [1-2, 4-10, 12]. The results of Björck [4] show that the problem of maximizing $S(n, m)$ for $\lambda<2$ is radically different from the problem for $\lambda>2$.

In the course of proving Theorem 1.1 we shall in fact prove a bit more. We say that a continuous and strictly increasing real valued function $f(x)$ defined for $0 \leqq x \leqq 2$ has the monotonic midpoint property for $\theta$ if the following is true: whenever $q$ is on an arc of length greater than or equal to $\theta$, with endpoints $q_{1}, q_{2} \in U$, the sum $f\left(\left|q-q_{1}\right|\right)+f\left(\left|q-q_{2}\right|\right)$ is maximal if and only if $q$ is the midpoint of this arc, and, moreover, the sum decreases strictly as $q$ moves away from the midpoint to $q_{1}$. Let $M(\theta)$ be the class of all such functions.

THEorem 1.3. For $p_{1}, p_{2}, p_{3} \in U$ and $f \in M(\pi)$ there is a $p \in U$ such that

$$
\sum_{i=1}^{3} f\left(\left|p-p_{i}\right|\right) \geqq \max _{p \in U} \sum_{i=1}^{3} f\left(\left|p-e_{i}\right|\right)
$$

where the $e_{i}$ are the vertices of an equilateral triangle. Equality holds if and only if $p_{1}, p_{2}, p_{3}$ are themselves the vertices of an equilateral triangle.

It is rather easy to show that $f(x)=x^{\lambda} \in M(0)$ for $0<\lambda \leqq 1$, and that for any $\theta>\pi$ it belongs to $M(\theta)$ when $\lambda=2$; it is true but somewhat more difficult to show that $f(x) \in M(\pi)$ when $1<\lambda<$ 2. We give the details in $\S 3$. Clearly Theorems 1.2 and 1.3 imply Theorem 1.1. The proof of Theorem 1.3 is given in $\S 5$, after the preliminary results of $\S 4$.

2. The regular $n$-gon. For $n \geqq 3$, let $e_{1}, \cdots, e_{n}$ be, in counterclockwise order, the vertices of a regular $n$-gon inscribed in $U$. Choose co-ordinates so that $p_{0}=(1,0)$ lies on the midpoint of arc $\left(e_{1}, e_{n}\right)$, the smaller arc determined by $e_{1}$ and $e_{n}$. We shall obtain Theorem 1.2 from the differential inequality of Lemma 2.3. Lemmas 2.1, 2.2, and 2.3 are probably obvious to any expert on Green functions, but we give complete proofs to keep the paper self-contained. 
Lemma 2.1. Assume that $f(x)$ has a continuous second derivative for $0<x<T$, that $f(0)=f(T)=0$, and that

$$
f^{\prime \prime}(x)+k^{2} f(x)=r(x)
$$

where $k$ is a constant such that $0<k T<\pi$. Then $f(x)=J(x)$, where

$$
J(x)=\int_{0}^{T} G(x, t) r(t) d t,
$$

and

(2.3) $\quad G(x, t)=\{\sin k T \sin k(x-t)-\sin k x \sin k(T-t)\} / k \sin k T$ for $0 \leqq t \leqq x \leqq T$, and

$$
G(x, t)=G(t, x)
$$

for $0 \leqq x \leqq t \leqq T$.

Proof. Since $G(x, t)$, as a function of $x$, need not be differentiable at $t$, we must use considerable care in verifying that $J(x)$ satisfies (2.1). Write

$$
\begin{aligned}
J(x) & =\int_{0}^{T} G(x, t) r(t) d t \\
& =\int_{0}^{x} G(x, t) r(t) d t+(-1) \int_{T}^{x} G(x, t) r(t) d t \\
& \equiv J_{1}(x)+J_{2}(x) .
\end{aligned}
$$

For $J_{1}(x)$ we can assume that (2.3) is always valid. Thus

$$
J_{1}^{\prime}(x)=G(x, x) r(x)+\int_{0}^{x} G_{x}(x, t) r(t) d t
$$

and

$$
J_{1}^{\prime \prime}(x)+k^{2} J_{1}(x)=\frac{d}{d x}\{G(x, x) r(x)\}+\left.G_{x}(x, t) r(t)\right|_{t=x}
$$

since $k^{2} G(x, t)+G_{x x}(x, t)=0$. For $J_{2}$ we can assume that

$$
G(x, t)=\{\sin k T \sin k(t-x)-\sin k t \sin k(T-x)\} / k \sin k T
$$

is always valid, so

$$
J_{2}^{\prime}(x)=-G(x, x) r(x)-\int_{T}^{x} G_{x}(x, t) r(t) d t
$$

and

$$
J_{2}^{\prime \prime}(x)+k^{2} J_{2}(x)=-\frac{d}{d x}\{G(x, x) r(x)\}-\left.G_{x}(x, t) r(t)\right|_{t=x} .
$$


Hence (remember that $G$ has a "two-part definition")

$$
\begin{aligned}
J^{\prime \prime}(x) & +k^{2} J(x) \\
= & \frac{r(x)}{\sin k T}\left\{\left.\sin k T \cos k(x-t)\right|_{t=x}-\left.\cos k x \sin k(T-t)\right|_{t=x}\right. \\
& \left.\quad+\left.\sin k T \cos k(t-x)\right|_{t=x}-\left.\sin k t \cos k(T-x)\right|_{t=x}\right\} \\
= & r(x)\left\{2-\frac{\sin (k x+k(T-x))}{\sin k T}\right\}=r(x) ;
\end{aligned}
$$

it is also clear that $J(0)=J(T)=0$. Now if $K(x)$ is another solution to (2.1) with $K(0)=K(T)=0$, then $L(x)=J(x)-K(x)=A \cos k x+$ $B \sin k x$ for certain constants $A$ and $B$. From $L(0)=L(T)=0$, it follows that $A=B=0$. This completes the proof.

Lemma 2.2. Under the hypotheses of Lemma 2.1 we have

$$
G(x, t) \leqq 0
$$

for $0 \leqq x, t \leqq T$.

Proof. It suffices to prove this for $0 \leqq t \leqq x \leqq T$. In that case

$$
G(x, t)=\left\{\sin ^{2} \theta_{1}-\sin ^{2} \theta_{2}\right\} / k \sin k T
$$

where

$$
-\frac{\pi}{2}<\theta_{1}=\frac{k(T-t-x)}{2} \leqq \theta_{2}=\frac{k(T-x+t)}{2}<\frac{\pi}{2} .
$$

Thus (consider the graph of $\sin ^{2} x$ for $-\pi / 2 \leqq x \leqq \pi / 2$ ) it suffices to show $\theta_{2}$ is closer to $\pi / 2$ than $\theta_{1}$ is to $-\pi / 2$. But $\theta_{1}-(-\pi / 2) \geqq \pi / 2-\theta_{2}$ is equivalent to $k T \geqq k x$, so the proof is complete.

Lemma 2.3. Assume that $f(x)$ has a continuous second derivative for $0<x<T$, that $f(0)=f(T)=0$, and that

$$
f^{\prime \prime}(x)+k^{2} f(x) \leqq 0
$$

where $k$ is a constant such that $0<k T<\pi$. Then $f(x)$ is a nonnegative concave function for $0 \leqq x \leqq T$.

Proof. Set $r(x)=f^{\prime \prime}(x)+k^{2} f(x)$. From (2.2) and Lemma 2.2 we see that $f(x) \geqq 0$ for $0 \leqq x \leqq T$. Hence $f^{\prime \prime}(x)=-k^{2} f(x)+r(x) \leqq$ 0 for $0 \leqq x \leqq T$ and the proof is complete.

Note that if the inequality (2.6) were reversed, the conclusion would be that $f(x)$ is nonpositive and convex.

We can now prove Theorem 1.2. Write $n=2 k+s$ where $k$ and $s$ are integers with $0 \leqq s \leqq 1$. We can assume without loss of 
generality that $p=(\cos \phi, \sin \phi)$ is on the smaller arc joining $e_{1}$ and $e_{n}$. Set

$$
\begin{aligned}
F_{\lambda}(\phi) \equiv & 2^{-\lambda} \sum_{i=1}^{n}\left|p-e_{i}\right|^{\lambda} \\
= & \sum_{j=1}^{k} \sin ^{\lambda}\left(\frac{(2 j-1) \pi}{2 n}-\frac{\dot{\phi}}{2}\right) \\
& +\sum_{j=1}^{k} \sin ^{\lambda}\left(\frac{(2 j-1) \pi}{2 n}+\frac{\phi}{2}\right)+s \sin ^{\lambda}\left(\frac{\pi}{2}-\frac{\phi}{2}\right) .
\end{aligned}
$$

Here the first sum on the right involves distances from $p$ to vertices strictly above the $x$-axis, the second sum is for vertices strictly below the $x$-axis, and the third term corresponds to the vertex, if any, at $(-1,0)$.

For $0<\lambda \leqq 1$ Theorem 1.2 is an easy consequence of the second part of Lemma 3.2. For $1<\lambda<2$, the second derivative of $F_{\lambda}(\dot{\phi})$ with respect to $\phi$ has singularities, so $F_{\lambda}(\phi)$ cannot be constant on any interval. Thus it suffices to show that $F^{\prime \prime}(\phi) \leqq 0$ for $0 \leqq \phi \leqq \pi / n$ where $F(\phi)=F_{\lambda}(\phi)$. Since $F(-\phi)=F(\phi)$ it then follows for $0 \leqq \phi \leqq$ $\pi / n$ that $F(\phi)$ is maximal if and only if $\phi=0$ and minimal if and only if $\phi=\pi / n$. Make the change of variable $\delta=\lambda-1$ and $t=$ $\pi / 2 n-\phi / 2$. Then

$$
\begin{aligned}
F(\dot{\phi})= & \sum_{j=1}^{k} \sin ^{1+o}\left(\frac{(j-1) \pi}{n}+t\right)+\sum_{j=1}^{k} \sin ^{1+o}\left(\frac{j \pi}{n}-t\right) \\
& +s \sin ^{1+o}\left(\frac{(n-1) \pi}{2 n}+t\right) .
\end{aligned}
$$

It now suffices to show that $K_{\dot{o}}(t) \geqq 0$ for $0 \leqq t \leqq \pi / 2 n$ and $-1<$ $\delta<1$ where

$$
\begin{aligned}
K_{\hat{o}}(t) \equiv & \frac{1}{1+\delta} \frac{d F(\phi)}{d t}=\sum_{j=1}^{k} \sin ^{o}\left(\frac{(j-1) \pi}{n}+t\right) \cos \left(\frac{(j-1) \pi}{n}+t\right) \\
& -\sum_{j=1}^{k} \sin ^{o}\left(\frac{j \pi}{n}-t\right) \cos \left(\frac{j \pi}{n}-t\right) \\
& +s \sin ^{o}\left(\frac{(n-1) \pi}{2 n}+t\right) \cos \left(\frac{(n-1) \pi}{2 n}+t\right) .
\end{aligned}
$$

If $\delta$ is negative, this follows from

$$
\frac{(j-1) \pi}{n}+t \leqq \frac{j \pi}{n}-t
$$

because each term of the second sum of (2.8) is then at most as large as the corresponding term of the first sum. Next, say $0 \leqq \delta \leqq 1$. It is easy to verify that the differential equation 


$$
J_{\delta}^{\prime \prime}(t)+(\delta+1)^{2} J_{\delta}(t)=-\delta(1-\delta) J_{\delta-2}(t)
$$

is satisfied by $J_{\delta}(t)=\sin ^{\delta} t \cos t$. Thus it is also satisfied by $K_{\delta}(t)$. Also, $K_{\delta-2}(t) \geqq 0$ since $\delta-2$ is negative. Moreover, $K_{\delta}(0)=K_{\delta}(\pi / 2 n)=$ 0 and $0<(\delta+1) \pi / 2 n<\pi$. Thus Lemma 2.3 yields $K_{\delta}(t) \geqq 0$ for $0 \leqq t \leqq \pi / 2 n$ and the proof is complete for $0<\lambda<2$.

Next, say $2<\lambda<3$, so $1<\delta<2$. Then (2.9), the fact that $\delta-2$ is negative, and Lemma 2.3 yield $K_{\delta}(t) \leqq 0$. For $2<\delta<3$ we have $K_{\delta-2}(t) \geqq 0$ by what has already been proved, and $-\delta(1-\delta)>$ 0 . Hence Lemma 2.3 once again yields $K_{\delta}(t) \leqq 0$. This completes the proof for $2<\lambda<4$. By continuing in this manner we obtain the theorem for $0<\lambda<2 n$ whenever $\lambda$ is strictly between even integers. But if $\lambda$ equals an even integer in this range, then it is clear by continuity that $T_{\lambda}(p)$ is constant.

3. The midpoint property. Let $f(x)=x^{\lambda}$ where $0<\lambda<2$. Our goal here is Lemma 3.2.

LEMMA 3.1. If $\delta<1$, then

$$
(\sin u)^{\delta} \cos u \leqq(\sin v)^{\delta} \cos v
$$

for $(u, v)$ in the isosceles triangle $T$ determined by $\pi / 2-u \leqq v \leqq u$ and $\pi / 4 \leqq u \leqq \pi / 2$. If $\delta \neq 0$, equality holds if and only if $u=v$ or $(u, v)=(\pi / 2,0)$. If $\delta=0$ equality holds if and only if $u=v$.

Proof. Inequality (3.1) is clearly true for $u=\pi / 2$ and $u=v$. For $u=\pi / 2-v$ the inequality becomes

$$
(\sin v)^{1-\delta} \leqq(\cos v)^{1-\delta}
$$

which is obviously true since $v \leqq \pi / 4$. Thus (3.1) is true on the boundary of $T$, so it suffices to show that

$$
f(u, v)=\delta \log \sin v+\log \cos v-\delta \log \sin u-\log \cos u
$$

has no critical point in the interior of $T$. At a critical point $(u, v)$, we have

$$
\frac{\partial f}{\partial u}=-\delta \cot u+\tan u=0
$$

and

$$
\frac{\partial f}{\partial v}=\delta \cot v-\tan v=0
$$

so $\delta=\tan ^{2} v=\tan ^{2} u$. Thus $u=v$ and $(u, v)$ is not in the interior of $T$. The cases of equality are trivially determined by inspecting (3.1) 
on the boundary of $T$. This completes the proof.

LEMMA 3.2. If $f(x)=x^{\lambda}$ where $0<\lambda<2$ then $f(x) \in M(\pi)$. If $0<\lambda \leqq 1$ then $f(x) \in M(0)$.

Proof. Let $q_{1}=(\cos \theta, \sin \theta), q_{2}=(\cos \theta,-\sin \theta)$, and $q=(\cos \phi$, $\sin \phi$ ) where $\pi / 2 \leqq \theta \leqq \pi$ and $0<\phi<\theta$. We must first show that $g^{\prime}(\phi)<0$ where

$$
\begin{aligned}
g(\phi) & =f\left(\left|q-q_{1}\right|\right)+f\left(\left|q-q_{2}\right|\right) \\
& =\left(2 \sin \frac{\theta-\phi}{2}\right)^{2}+\left(2 \sin \frac{\theta+\phi}{2}\right)^{2}
\end{aligned}
$$

i.e., that

$$
\left(\sin \frac{\theta+\phi}{2}\right)^{\lambda-1} \cos \frac{\theta+\phi}{2}<\left(\sin \frac{\theta-\phi}{2}\right)^{\lambda-1} \cos \frac{\theta-\phi}{2} .
$$

In the case where $\pi-\theta \leqq \phi$ it is clear geometrically that increasing $\phi$ will strictly decrease both $\left|q-q_{1}\right|$ and $\left|q-q_{2}\right|$. If $\phi \leqq \pi-\theta$, make the change of variables $u=(\theta+\phi) / 2$ and $v=(\theta-\phi) / 2$. Since $\lambda-1<1$, the first statement follows from Lemma 3.1.

To prove the second statement, assume $0<\phi<\theta \leqq \pi$. Again we can assume $\phi \leqq \pi-\theta$. But then

$$
0<\frac{\theta-\phi}{2}<\frac{\theta+\phi}{2} \leqq \frac{\pi}{2}
$$

and, since $0<\lambda \leqq 1$, inequality (3.2) is immediate. This completes the proof.

4. Lemmas on maximality. The first two results of this section are almost self-evident, but we prove them for the sake of completeness.

LeMma 4.1. Let $X$ and $Y$ be compact subsets of an m-dimensional Euclidean space and let $F$ be a real valued continuous function on $X \times Y$. Define $G(y)$ by

$$
G(y)=\max _{x \in X} F(x, y) .
$$

Then $G(y)$ is a continuous function of $y$.

Proof. If not, let $\left\{y_{n}\right\}$ be an infinite sequence converging to some $y \in Y$ such that $\left|G\left(y_{n}\right)-G(y)\right|>c>0$ for some fixed positive constant c. Since $X$ is compact we can choose $x_{n}$ so that $G\left(y_{n}\right)=F\left(x_{n}, y_{n}\right)$. 
Since $X \times Y$ is compact we can choose a subsequence so that $\left(x_{n}, y_{n}\right) \rightarrow$ $\left(x^{\prime}, y\right)$ for some $x^{\prime} \in X$. Finally, choose a subsequence again so that either $G\left(y_{n}\right)>G(y)+c$ or $G(y)>G\left(y_{n}\right)+c$ for all $n$. Since $F$ is continuous

$$
G\left(y_{n}\right)=F\left(x_{n}, y_{n}\right) \longrightarrow F\left(x^{\prime}, y\right) \leqq G(y)=F\left(x^{\prime \prime}, y\right)
$$

for some $x^{\prime \prime} \in X$. Hence $G(y)>G\left(y_{n}\right)+c$. Also

$$
G\left(y_{n}\right) \geqq F\left(x^{\prime \prime}, y_{n}\right) \longrightarrow F\left(x^{\prime \prime}, y\right)=G(y),
$$

so $G\left(y_{n}\right)>G(y)+c$. This contradiction proves the Lemma.

LEMMA 4.2. If

$$
K=K\left(p_{1}, p_{2}, p_{3}\right)=\max _{p \in U} \sum_{i=1}^{3} f\left(\left|p-p_{i}\right|\right)
$$

where $f$ is continuous, $U$ is the unit circle, and $p_{1}, p_{2}$, and $p_{3}$ are points of $U$, then $K$ is continuous.

Proof. Apply Lemma 4.1 with $X=U, Y=U \times U \times U$, and

$$
F(p)=\sum_{i=1}^{3} f\left(\left|p-p_{i}\right|\right)
$$

Before stating Lemma 4.3, we introduce some terminology. If $a_{1}, a_{2}$, and $a_{3}$ are three points on $U$, we denote by arc $\left(a_{1}, a_{2}\right)$ that closed arc, with $a_{1}$ and $a_{2}$ as its endpoints, which does not contain $a_{3}$. We define $\operatorname{arc}\left(a_{1}, a_{3}\right)$ and $\operatorname{arc}\left(a_{2}, a_{3}\right)$ similarly.

LEMMA 4.3. Let

$$
F(p)=F\left(p ; p_{1}, p_{2}, p_{3}\right)=\sum_{i=1}^{3} f\left(\left|p-p_{i}\right|\right),
$$

where $f$ is continuous and strictly increasing. Then $F(p)$ is a continuous function on $U$ which attains its maximum on every longest arc of the triple $\left\{\operatorname{arc}\left(p_{1}, p_{2}\right)\right.$, $\left.\operatorname{arc}\left(p_{1}, p_{3}\right), \operatorname{arc}\left(p_{2}, p_{3}\right)\right\}$, and nowhere else.

Proof. We shall prove only the case where one of the arcs (call it $\alpha$ ) is strictly larger than the others; the rest of the argument is quite similar. If the length of $\alpha$ is at least $\pi$, let $d$ be a diameter of $U$ such that $\alpha$ contains both endpoints of $d$. If $F$ has a maximum at $p$ and $p \notin \alpha$, let $p^{\prime}$ be the reflection of $p$ in $d$. Clearly $F\left(p^{\prime}\right)>$ $F(p)$, a contradiction. Thus we can assume that the length of $\alpha$ is less than $\pi$. Say $\alpha=\operatorname{arc}\left(p_{1}, p_{2}\right)$, but $F(p)$ is maximal for some 
$p \in \operatorname{arc}\left(p_{3}, p_{1}\right)$. Choose $p^{\prime} \in \operatorname{arc}\left(p_{1}, p_{2}\right)$ so that $\left|p_{2}-p^{\prime}\right|=\left|p_{3}-p\right|$. It follows that $\left|p-p_{2}\right|=\left|p^{\prime}-p_{3}\right|$ and $\pi \geqq \operatorname{arc}\left(p^{\prime}, p_{1}\right)>\operatorname{arc}\left(p, p_{1}\right) \geqq 0$, so $\left|p^{\prime}-p_{1}\right|>\left|p-p_{1}\right|$. Hence $F\left(p^{\prime}\right)>F(p)$. This contradiction proves the result.

LEMMA 4.4. Let $p_{1}, p_{2}$, and $p_{3}$ be points on $U$. Let $L$ be a line parallel to seg $\left(p_{1}, p_{2}\right)$, the line segment joining $p_{1}$ and $p_{2}$. Assume $L$ separates seg $\left(p_{1}, p_{2}\right)$ from $p_{3}$, and let $p_{1}^{\prime}, p_{2}^{\prime}$ be the points at which $L$ intersects $U$. Assume that the smallest arc between $p_{3}$ and $p_{m}$ is at least $\pi / 2$, where $p_{m}$ is the midpoint of $\operatorname{arc}\left(p_{1}, p_{2}\right)$. Then for $f \in M(\pi)$ we have

$$
\begin{aligned}
a & \equiv f\left(\left|p_{1}-p_{3}\right|\right)+f\left(\left|p_{2}-p_{3}\right|\right) \\
& \geqq f\left(\left|p_{1}^{\prime}-p_{3}\right|\right)+f\left(\left|p_{2}^{\prime}-p_{3}\right|\right) \equiv b
\end{aligned}
$$

with equality if and only if $p_{1}=p_{1}^{\prime}$ and $p_{2}=p_{2}^{\prime}$.

Proof. Let $l$ be the line joining $p_{m}$ and the center of $U$. Let $p_{3}^{\prime}$ be the reflection of $p_{3}$ in $l$. Since $f \in M(\pi)$ we have

$$
\begin{aligned}
2 a & =\left[f\left(\left|p_{1}-p_{3}\right|\right)+f\left(\left|p_{1}-p_{3}^{\prime}\right|\right)\right]+\left[f\left(\left|p_{2}-p_{3}\right|\right)+f\left(\left|p_{2}-p_{3}^{\prime}\right|\right)\right] \\
& \geqq\left[f\left(\left|p_{1}^{\prime}-p_{3}\right|\right)+f\left(\left|p_{1}^{\prime}-p_{3}^{\prime}\right|\right)\right]+\left[f\left(\left|p_{2}^{\prime}-p_{3}\right|\right)+f\left(\left|p_{2}^{\prime}-p_{3}^{\prime}\right|\right)\right] \\
& =2 b
\end{aligned}
$$

with equality if and only if $p_{1}=p_{1}^{\prime}$ and $p_{2}=p_{2}^{\prime}$.

5. The proof. Let the continuous (by Lemma 4.2) function $K\left(p_{1}, p_{2}, p_{3}\right)$ have its absolute minimum at $\left(p_{1}, p_{2}, p_{3}\right)=\left(P_{1}, P_{2}, P_{3}\right)$. It is easy to show that no $\operatorname{arc}\left(P_{i}, P_{j}\right)$ is longer than $\pi$. If some arc, say $\operatorname{arc}\left(P_{1}, P_{2}\right)$, is strictly larger than the others, then there is a $P_{2}^{\prime}$ such that $\operatorname{arc}\left(P_{1}, P_{2}^{\prime}\right)$ is larger than $\operatorname{arc}\left(P_{2}^{\prime}, P_{3}\right)$ and $\operatorname{arc}\left(P_{3}, P_{1}\right)$, but smaller than $\operatorname{arc}\left(P_{1}, P_{2}\right)$. Now, by Lemma 4.3 , the function $F\left(p ; P_{1}\right.$, $\left.P_{2}^{\prime}, P_{3}\right)$ will attain its maximum at some $p_{0} \in \operatorname{arc}\left(P_{1}, P_{2}^{\prime}\right)$. Since $\mid p_{0}$ $P_{2}^{\prime}|<| p_{0}-P_{2} \mid$, we have

$$
\begin{aligned}
K\left(P_{1}, P_{2}^{\prime}, P_{3}\right) & =F\left(p_{0} ; P_{1}, P_{2}^{\prime}, P_{3}\right) \\
& <F\left(p_{0} ; P_{1}, P_{2}, P_{3}\right) \leqq K\left(P_{1}, P_{2}, P_{3}\right),
\end{aligned}
$$

a contradiction. Hence we may assume that

$$
\frac{2 \pi}{3} \leqq \operatorname{arc}\left(P_{1}, P_{3}\right)=\operatorname{arc}\left(P_{2}, P_{3}\right) \geqq \operatorname{arc}\left(P_{1}, P_{2}\right) .
$$

If strict inequality holds, let $L$ be a line parallel to seg $\left(P_{1}, P_{2}\right)$ which separates it from $P_{3}$, and so that

$$
\operatorname{arc}\left(P_{1}^{\prime}, P_{3}\right)=\operatorname{arc}\left(P_{2}^{\prime}, P_{3}\right)>\operatorname{arc}\left(P_{1}^{\prime}, P_{2}^{\prime}\right)
$$


where $P_{1}^{\prime}$ and $P_{2}^{\prime}$ are the points of intersection of $L$ with $U$. Now by Lemma 4.3 , the function $F\left(p ; P_{1}^{\prime}, P_{2}^{\prime}, P_{3}\right)$ will attain its maximum at some $p_{0} \in \operatorname{arc}\left(P_{1}^{\prime}, P_{3}\right)$. If $\operatorname{arc}\left(p_{0}, P_{m}\right)<\pi / 2$ where $P_{m}$ is the midpoint of $\operatorname{arc}\left(P_{1}, P_{2}\right)$, choose $p_{0}^{\prime} \in \operatorname{arc}\left(P_{3}, P_{1}^{\prime}\right)$ so that $\left|p_{0}^{\prime}-P_{3}\right|=\left|p_{0}-P_{1}^{\prime}\right|$. Then $\left|p_{0}^{\prime}-P_{1}^{\prime}\right|=\left|p_{0}-P_{3}\right|$ and $\left|p_{0}^{\prime}-P_{2}^{\prime}\right| \geqq\left|p_{0}-P_{2}^{\prime}\right|$ (consider the perpendicular bisector of $\left.\operatorname{seg}\left(p_{0}, p_{0}^{\prime}\right) !\right)$. Hence we can assume that $\operatorname{arc}\left(p_{0}, P_{m}\right) \geqq \pi / 2$. By Lemma 4.4 we now have that

$$
\begin{gathered}
f\left(\left|p_{0}-P_{1}^{\prime}\right|\right)+f\left(\left|p_{0}-P_{2}^{\prime}\right|\right)+f\left(\left|p_{0}-P_{3}\right|\right)<f\left(\left|p_{0}-P_{1}\right|\right) \\
+f\left(\left|p_{0}-P_{2}\right|\right)+f\left(\left|p_{0}-P_{3}\right|\right),
\end{gathered}
$$

so

$$
\begin{aligned}
K\left(P_{1}^{\prime}, P_{2}^{\prime}, P_{3}\right) & =F\left(p_{0} ; P_{1}^{\prime}, P_{2}^{\prime}, P_{3}\right) \\
& <F\left(p_{0} ; P_{1}, P_{2}, P_{3}\right) \leqq K\left(P_{1}, P_{2}, P_{3}\right),
\end{aligned}
$$

a contradiction. Hence all arcs are equal and $P_{1}, P_{2}$, and $P_{3}$ must be the vertices of an equilateral triangle. This completes the proof of Theorem 1.3. Theorem 1.1 is an easy consequence.

6. REMARK. In view of Lemma 3.2 , it is quite easy to show (in the notation of Theorem 1.1) that there is a $p \in U$ such that

$$
\sum_{i=1}^{3}\left|p-p_{i}\right|^{\lambda} \leqq 2 \cdot 3^{\lambda / 2}
$$

That this is best possible again follows from Theorem 1.2. However, the problem of generalizing Theorem 1.1 to the case of $n$ points $p_{1}$, $p_{2}, \cdots, p_{n}$ may require new ideas for its solution. We also mention that the proof of Theorem 1.2 (see (2.8)) shows incidentally that for $\lambda<0$ the sum $T_{\lambda}(p)$ is minimal if and only if $p$ bisects the arc between consecutive $e_{i}$. However, to determine the extremal behavior of $T_{\lambda}(p)$ for $\lambda \geqq 2 n$ seems to require a different approach.

\section{REFERENCES}

1. J. Ralph Alexander, On the sum of distances between $n$ points on a sphere, Acta Math. Acad. Sci. Hungar., 23 (1972), 443-448.

2. J. Ralph Alexander and K. B. Stolarsky, Extremal problems of distance geometry related to energy integrals, Trans. Amer. Math. Soc., 193 (1974), 1-31.

3. E. F. Beckenbach and R. Bellman, Inequalities, Springer-Verlag, Berlin, 1971 (third printing).

4. G. Björck, Distributions of positive mass which maximize a certain generalized energy integral, Ark. Mat., 3 (1955), 255-269.

5. G. D. Chakerian and M. S. Klamkin, Inequalities for sums of distances, Amer. Math. Monthly, 80 (1973), 1009-1017.

6. L. Fejes Toth, On the sum of distances determined by a pointset, Acta Math. Acad.

Sci. Hungar., 7 (1956), 397-401. 
7. E. Hille, Some geometric extremal problems, J. Australian Math. Soc., 6 (1966), 122-128.

8. G. Polyá and G. Szegö, Über den transfiniten Durchmesser (Kapazitätskonstante) von ebenen und räumlichen Punktmengen, J. Reine Angew. Math., 165 (1931), 4-49.

9. K. B. Stolarsky, Sums of distances between points on a sphere, Proc. Amer. Math. Soc., 35 (1972), 547-549.

10. Sums of distances between points on a sphere II, Proc. Amer. Math. Soc., 41 (1973), 575-582.

11. - The sum of the distances to $N$ points on the unit sphere, Pacific J. of Math., to appear.

12. D. Wolfe, Metric dependence and a sum of distances, in preparation.

Received March 19, 1975.

UNIVERSITY OF COLORADO

AND

UNIVERSITY OF ILLINOIS 



\section{PACIFIC JOURNAL OF MATHEMATICS}

\section{EDITORS}

RICHARD ARENS (Managing Editor) University of California

Los Angeles, California 90024

\section{R. A. Beaumont}

University of Washington Seattle, Washington 98105
J. DugundJI Department of Mathematics University of Southern Californıa Los Angeles, California 90007

D. Gilbarg aNd J. Milgram Stanford University Stanford, California 94305

\section{ASSOCIATE EDITORS}

E. F. BeCKENBACH

B. H. NeUmanN

F. Wolf

K. YoSHIDA

\section{SUPPORTING INSTITUTIONS}

UNIVERSITY OF BRITISH COLUMBIA CALIFORNIA INSTITUTE OF TECHNOLOGY UNIVERSITY OF CALIFORNIA MONTANA STATE UNIVERSITY UNIVERSITY OF NEVADA NEW MEXICO STATE UNIVERSITY OREGON STATE UNIVERSITY UNIVERSITY OF OREGON OSAKA UNIVERSITY
UNIVERSITY OF SOUTHERN CALIFORNIA STANFORD UNIVERSITY UNIVERSITY OF TOKYO UNIVERSITY OF UTAH WASHINGTON STATE UNIVERSITY UNIVERSITY OF WASHINGTON AMERICAN MATHEMATICAL SOCIETY NAVAL WEAPONS CENTER 


\section{Pacific Journal of Mathematics \\ Vol. 59, No. 1 \\ May, 1975}

Shashi Prabha Arya and M. K. Singal, More sum theorems for topological

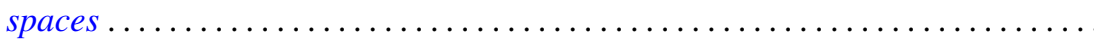

Goro Azumaya, F. Mbuntum and Kalathoor Varadarajan, On M-projective and

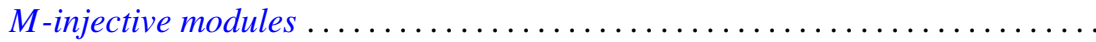

Kong Ming Chong, Spectral inequalities involving the infima and suprema of

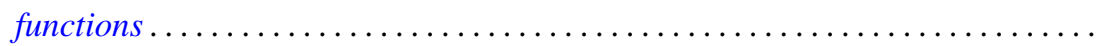

Alan Hetherington Durfee, The characteristic polynomial of the monodromy .......

Emilio Gagliardo and Clifford Alfons Kottman, Fixed points for orientation

preserving homeomorphisms of the plane which interchange two points ......

Raymond F. Gittings, Finite-to-one open maps of generalized metric spaces .......

Andrew M. W. Glass, W. Charles (Wilbur) Holland Jr. and Stephen H. McCleary,

$a^{*}$-closures of completely distributive lattice-ordered groups .............

Matthew Gould, Endomorphism and automorphism structure of direct squares of

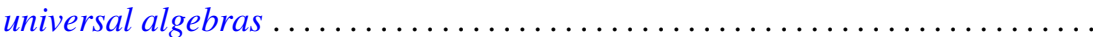

R. E. Harrell and Les Andrew Karlovitz, On tree structures in Banach spaces .....

Julien O. Hennefeld, Finding a maximal subalgebra on which the two Arens

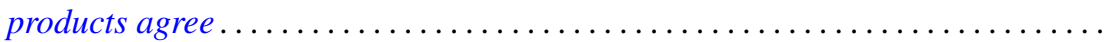

William Francis Keigher, Adjunctions and comonads in differential algebra .......

Robert Bernard Kelman, A Dirichlet-Jordan theorem for dual trigonometric

series

Allan Morton Krall, Stieltjes differential-boundary operators. III. Multivalued operators-linear relations...

Hui-Hsiung Kuo, On Gross differentiation on Banach spaces .

Tom Louton, A theorem on simultaneous observability ...

Kenneth Mandelberg, Amitsur cohomology for certain extensions of rings of algebraic integers.

Coy Lewis May, Automorphisms of compact Klein surfaces with boundary . . .

Peter A. McCoy, Generalized axisymmetric elliptic functions .

211

Muril Lynn Robertson, Concerning Siu's method for solving $y^{\prime}(t)=F(t$, $y(g(t)))$. .

Richard Lewis Roth, On restricting irreducible characters to normal

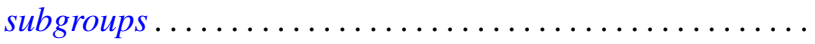

Albert Oscar Shar, $P$-primary decomposition of maps into an $H$-space .

Kenneth Barry Stolarsky, The sum of the distances to certain pointsets on the unit circle.

Bert Alan Taylor, Components of zero sets of analytic functions in $C^{2}$ in the unit ball or polydisc

Michel Valadier, Convex integrands on Souslin locally convex spaces ...

Januario Varela, Fields of automorphisms and derivations of $C$

Arnold Lewis Villone, A class of symmetric differential operators with deficiency

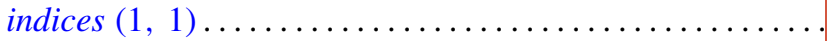

